\title{
Hepatitis B and hepatocellular carcinoma screening practices in Chinese and African immigrant-rich neighborhoods in New York City
}

\author{
S Fitzgerald, J Chao, Y Fefermen, P Perumalswami, and U Sarpel
}

\section{Background}

Hepatocellular carcinoma (HCC) is the second leading cause of cancer death worldwide, with approximately $80 \%$ of cases occurring in developing countries [1,2]. Chronic hepatitis $B$ virus (HBV) infection is the most common cause of HCC; in HBV endemic areas such as sub-Saharan Africa, West Africa, and China 70-90\% of HCC cases are attributable to HBV $[2,3]$. Many of the deaths from HBV-related HCC are preventable with treatment of HBV and earlier diagnosis of HCC [3]. However, despite the availability of highly effective antiviral regimens, HBV testing and treatment remain low for immigrants from these regions. A recent report commissioned by the Institute of Medicine emphasized the tremendous burden of viral hepatitis in the United States (U.S.) and the inadequacy of current screening efforts, lending urgency to the need for improved HBV testing and treatment strategies[4]. Guidelines for HBV testing of those from high-risk regions of Africa and Asia have been upgraded by the U.S. Preventive Services Task Force to a Grade B recommendation, indicating the presence of strong evidence to support its benefit [5].

Treatment of HBV has been shown to decrease the incidence of HCC, and regular screening for HCC among high-risk HBV patients has been shown to improve disease related mortality $[6,7]$. For this reason, current guidelines from the American Association for the Study of Liver Disease (AASLD) recommend HBV testing for all immigrants, as well as routine HCC screening by abdominal ultrasound every 6 months in high risk HBV carriers. It is recommended that $\mathrm{HCC}$ screening be performed for all Asian men older than 40 years and Asian women older than 50 years [8, 9]. Since African patients with HBV develop HCC at a younger age $[10,11]$, earlier AASLD guidelines had suggested that HCC screening begin at 20 years of age[9]. Current guidelines no longer provide an exact age, but suggest that African HBV carriers should be screened for HCC at an earlier age than other groups[8]. Despite the presence of these widely disseminated guidelines, adherence to recommended screening remains low among primary care providers (PCP).

\footnotetext{
Conflict of Interest: Authors Fitzgerald, Chao, Perumalswami, Feferman, and Sarpel all declare that they have no conflict of interest. Ethical approval: All procedures performed in studies involving human participants were in accordance with the ethical standards of the institutional and/or national research committee and with the 1964 Helsinki declaration and its later amendments or comparable ethical standards. Please note this study was determined to have "Exempt" status after formal consideration by our Institutional Review Board.
} 
As a result, culturally-targeted screening programs have been created in immigrant-rich cities such as New York City (NYC) and San Francisco. In these cities, large, well-organized screening programs have been established to target Chinese, Korean, and other Asian immigrant communities, but few have focused on African communities [12-17]. HBV is endemic in many parts of Africa, with a prevalence estimated between 8-20\% with similar prevalence reported amongst Africans living in the U.S. [18-20]. Furthermore, African immigration has increased more than $750 \%$ over the past 20 years, with 1.6 million Africans currently living in the U.S., making HBV screening of African-born patients and HCC screening among patients who test positive for $\mathrm{HBV}$ an important current issue in preventative medicine [21]. While the population of Chinese-born individuals in NYC is estimated to be more than 350,000 , the African immigrant population in NYC is still sizeable at approximately 132,000 people, representing $4 \%$ of foreign-born population in the city [22].

Thus, the primary aim of this study was to examine HBV and HCC knowledge, screening practices, and barriers to screening among PCPs in NYC treating immigrants from high-risk regions. The secondary aim was to compare the responses from neighborhoods with a high density of African versus Chinese immigrants, to determine whether differences existed by community. These data may inform the development of future targeted interventions to improve screening practices for at-risk immigrant patient populations.

\section{Methods}

This was a targeted, cross-sectional survey of PCPs who practice in the NYC zip codes with the greatest population of African or Chinese-born individuals. An exemption was obtained from our Institutional Review Board based on the anonymous nature of the survey.

\section{Participants}

The Population Division of the New York City Department of City Planning provided the NYC Neighborhood Tabulation Areas (NTA) with the highest numbers of Chinese and African-born individuals, based on the 2010 United States census data, as available on the NYC Census Fact Finder site [23]. These data represented the overall population for each site, including individuals of all ages.

Using the American Medical Association (AMA) New York State registry, physicians practicing within the zip codes that comprise each NTA were identified. The AMA Physician Masterfile is a commercial database which includes records on 1.4 million active and deceased physicians, residents, and medical students in the United States. This database was queried for practicing physicians meeting the following conditions: 1) Type of practice: Office Based or Hospital Staff; 2) Primary Specialty: Family Practice, General Practice, or Internal Medicine; and 3) Zip Code within the targeted neighborhood tabulation areas. Due to the email-based distribution of the survey, only physicians with an e-mail address on file were eligible. A total of 2,108 records were returned, of these 2,072 had a functional email address and thus comprised the study cohort. 
An email was sent to all physicians inviting them to participate, along with a link to the online survey asking for their voluntary participation. Due to a low response rate, we also contacted chairs of medicine at the major academic medical institutions within those zip codes to assist in distribution and promotion of the survey. No incentives (monetary or other) were used.

\section{Survey Development}

The online study survey instrument was composed of 28 questions and was developed by the study investigators based on reviews of previously published survey studies in the field [12-16, 24-26]. Survey content domains included provider and practice characteristics, provider knowledge of HBV and HCC screening and management practices, and barriers to screening. The survey was pilot tested with leaders serving the populations of interest to ensure clarity and cultural appropriateness of the questions; drawing from a diverse pool of public health advocates, community health center leaders and primary care physicians. Their suggestions were incorporated into the final iteration of the survey (Supplemental Material $1)$.

\section{Statistical Analysis}

The zip codes of responding providers were used to classify providers into the neighborhoods with the top 25 highest Chinese and African populations. In addition to descriptive statistics of the entire cohort, univariate analyses were conducted to compare the two communities, using the student t-test to compare continuous variables, and a chi-square test to compare categorical variables. Fisher exact tests were used to compare smaller populations where appropriate. $P$ values $0<.05$ (two-tailed) were considered to be statistically significant.

\section{Results}

The 25 neighborhoods with the greatest population of Chinese immigrants and the 25 neighborhoods with the greatest population of African immigrants included one overlapping area, for a total of 49 unique neighborhoods (Figure 1). The neighborhoods with the largest Chinese populations did not include any in the Bronx or Staten Island, but did include three in Manhattan, 11 in Brooklyn, and 11 in Queens. The neighborhoods with the largest African populations were spread out across the five boroughs of NYC and included 12 in the Bronx, three in Manhattan, seven in Brooklyn, two in Staten Island and one in Queens. The neighborhoods with the greatest Chinese immigrant populations contained 3,665-23,089 Chinese-born inhabitants per NTA, while the neighborhoods with the greatest African immigrant populations contained 1,308-3,505 African-born inhabitants (Figure 2). Thus, the Chinese community was more densely clustered than the African community in NYC.

Using the AMA database and directed emails, 2,072 physicians were contacted. A total of 109 physicians responded by filling out the questionnaire, for a response rate of 5.3\%, consistent with the stated typical response patters for this commercial database. The responders included 30 practicing in the 25 target NTAs for Chinese-born individuals, and 36 practicing in the target NTAs for African-born individuals. We received responses from 
24 physicians who provided a zip code outside of the target areas. In addition we received responses from 21 individuals who declined to provide the zip code of their practice location. Not every question was answered by each respondent, leading to varying denominators for the responses below.

\section{Practice Characteristics}

PCPs who responded to this question $(\mathrm{n}=85)$ reported having been in practice following completion of residency or fellowship for: less than 5 years (38\%); 5-10 years (16\%); 11-20 years $(26 \%)$; or greater than 20 years $(20 \%)$. The majority $(81 \%)$ stated that their practice was affiliated with an academic medical center. These values were not significantly different between the Chinese and African neighborhoods.

\section{English fluency and provider ethnicity}

Among all respondents ( $\mathrm{n}=99), 65 \%$ of the providers in Chinese neighborhoods, compared to $25 \%$ in the African neighborhoods reported the majority of their patients preferred to speak a language other than English. Thus, the providers in the Chinese neighborhoods were more likely to have patient populations that were primarily speakers of a foreign language $(\mathrm{p}=0.002)$. Of note, among respondents who provided their race $(\mathrm{n}=86), 53 \%$ of the providers practicing in the Chinese neighborhoods were themselves Asian ( $\mathrm{p}=0.003)$, and $41 \%$ of the providers in the African neighborhoods identified as black or African American $(\mathrm{p}=0.004)$.

\section{Insurance coverage}

Among respondents ( $\mathrm{n}=99$ ), providers in the neighborhoods with a greater Chinese immigrant population were more likely to have patient populations that were uninsured, with $76 \%$ responding that at least $10 \%$ of their patients lacked insurance, compared to the African-rich neighborhoods where $61 \%$ of providers reported at least $10 \%$ of their patients lacked insurance $(\mathrm{p}=0.027)$.

\section{Health concerns of patients}

PCPs reported that their patients' greatest health concerns were, in order of magnitude: diabetes, hypertension, heart disease, hepatitis B, HIV, obesity, kidney disease, other heath concern, liver cancer, colon cancer, and lung cancer (Fig.3). Thus neither HBV nor HCC was listed among the top three health concerns of patients, as perceived by providers in either of the surveyed communities. There was a significantly greater reporting of HIV as a health concern in the African compared to the Chinese immigrant communities $(\mathrm{p}=0.028)$.

\section{Screening practices}

Regarding screening, $73 \%$ of 99 responding providers report routinely testing immigrant patients for HBV. However, if a patient tests positive for HBV, only $68 \%$ of 93 providers answering this question recommend screening for HCC. Of 85 respondents, $78 \%$ correctly responded that screening for HCC should be performed for HBV-infected immigrant patients from high-risk regions, even if no symptoms are present. In addition, a large majority (92\%) 
correctly answered that $\mathrm{HCC}$ can occur in $\mathrm{HBV}$ infected patients without the presence of cirrhosis (Fig. 4).

Providers overwhelmingly correctly identified that ultrasound was the screening modality of choice for HCC (92\%), but only $64 \%$ correctly identified that ultrasound screening should be performed every 6-12 months, and only 56\% correctly replied that screening for liver cancer improves survival. In identifying the age at which high-risk patients who should begin HCC screening, 54\% correctly believed a 25 year-old patient from Africa with HBV should be screened, whereas $53 \%$ thought that a 25 year-old from China with HBV should be screened, demonstrating a lack of awareness of the different age of onset of HCC in the two groups (Fig.4). There was no statistically significant difference in PCP responses for provider knowledge between Chinese and African neighborhoods.

\section{Antiviral treatment}

PCPs were asked whether they prescribed antiretroviral treatment for those patients who tested positive for hepatitis B with treatment indicated. Among 71 respondents, $28 \%$ stated that they prescribed treatment themselves, $44 \%$ stated that they referred the patient to a hepatologist, $18 \%$ referred to a gastrointestinal specialist, and $7 \%$ referred to an infectious diseases specialist. Of note, over a quarter of PCPs (27\%), failed to correctly state that antiviral therapy can lower the risk of developing HCC.

\section{Provider barriers to screening}

The most common barriers for offering HBV testing among 65 respondents was "lack of clear screening guidelines" (49\%), with rates similar in both neighborhoods with large African and Chinese immigrant populations (Figure 5). "Competing health care priorities" (45\%) and "time constraints" (35\%) were other frequently reported barriers to care (Fig.4). Similar barriers were provided for HCC screening among the 59 responders to this question, with $61 \%$ again citing a "lack of clear screening guidelines". In addition, $32 \%$ of respondents felt there was a lack of referral options for patients found to have liver cancer.

\section{Patient barriers to screening}

When PCPs were asked to report what reasons their patients provided for not completing screening when recommended, respondents $(\mathrm{n}=81)$ reported that "lack of patients' awareness of hepatitis and liver cancer risk" (85\%), and "lack of insurance or cost to the patient" $(70 \%)$ as the main barriers faced by patients. There was no statistically significant difference in reported barriers between providers in the Chinese and African immigrant neighborhoods.

\section{Discussion}

HBV and HCC disproportionately affect Chinese and African immigrant patients [1, 27, 28]. PCP recommendations have been shown to improve hepatitis vaccination and cancer screenings among immigrant populations[17, 29], making the role of the provider tremendously important to the public health of these high- risk communities[6, 15]. Yet, to our knowledge, this is the first study to assess PCP knowledge, attitudes and behavior with 
respect to $\mathrm{HBV}$ and $\mathrm{HCC}$ screening in neighborhoods with significant African and Asian immigrant populations in NYC.

While the neighborhoods with large African and Chinese immigrant populations are spread out across the five boroughs of NYC, the Chinese communities are much more densely populated than the African communities. The providers in these neighborhoods were often themselves of the same ethnicity as their population served. This is important since Asian providers have been shown to more likely screen for HBV and HCC in Asian American communities $[12,13,30]$. Similarly, practices with more than $25 \%$ Asian immigrant patients have previously been shown to be more likely to screen for HCC[30] in HBV infected patients.

Within this study, providers in all neighborhoods report that their patients' greatest health concerns were diabetes, hypertension and heart disease. While similar numbers reported $\mathrm{HBV}$ as a concern, this was of lower concern than HIV in the African immigrant neighborhoods, suggesting that the African immigrant community's concern for HIV risks out-competes HBV and HCC for patients' healthcare priorities in a way that does not appear to happen in Chinese communities.

In our study, almost one-third of physicians reported that they did not routinely recommend HCC screening in those patients who test positive for HBV. If our results are generalizable, this suggests that at least one-third of high-risk HBV positive immigrant patients that should be screened for HCC are not being tested. Indeed, true screening rates are likely to be even lower than those self-reported by responders in this survey. This demonstrates a significant need for HCC screening improvement in high-risk immigrant neighborhoods.

Though previous studies of African immigrant communities are lacking, the reported screening practices in this survey are similar to previous studies of screening in AsianAmerican communities. Among providers in San Francisco, 70-76\% reported regular screening of HBV and 79-96\% reported regular HCC surveillance of HBV infected patients. However, a chart review of Asian patients for whom screening was recommended, found only $63 \%$ had any screening for HCC in the previous year, and two-thirds of such patients had never had abdominal imaging for HCC as per guidelines [16, 30]. A chart review of over 800 Chinese-speaking patients found that only $65 \%$ of patients had been appropriately screened for HBV [25]. Another survey of physicians in California found $84-88 \%$ of practitioners regularly screened high risk patients with HBV for HCC [26].

In our study, there was evidence of significant gaps in provider knowledge. Less than twothirds correctly identified that an ultrasound every 6-12 months was the recommended method of screening for HCC. Perhaps most alarming, over a quarter of PCPs were unaware that antiviral therapy can lower a patient's risk of developing HCC, and barely half were aware that HCC screening has been shown to improve survival among HBV positive patients. Previous research has shown that HCC screening is effective in improving HCC related mortality[6], and that recognition of this fact among providers improves screening rates[26]. Improved education of providers about the proven benefits of HCC screening among such providers to high-risk patients could have substantial benefits. 
Overall, these knowledge responses are similar to previous research showing that providers surveyed endorsed $71 \%$ of current CDC guidelines for HBV vaccination guidelines [31]. Another survey found $73 \%$ of providers answered HBV knowledge questions correctly, but that only $56 \%$ of these providers were familiar with AASLD guidelines for HBV management[30]. Higher rates of safety net providers of Asian Americans (70\%) were found to recognize the value of HCC screening to decrease HCC mortality[16].

The most common barrier cited by providers for providing either HBV or HCC screening was lack of clear guidelines, and this was similar in both African and Chinese neighborhoods. This is surprising given the presence of validated and well-accepted guidelines [8]. There was also a clear lack of awareness of the different age of onset of HCC in Asian versus African immigrants. Until recently, national guidelines recommended HCC screening of African patients with HBV beginning at age 20 [9]. However, guidelines have become less precise, and this may be a reason that almost $60 \%$ of providers who responded from neighborhoods with significant African populations feel that the lack of clear guidelines are a barrier to offering HCC screening in their immigrant patients with HBV.

The conclusions that can be drawn from this study are limited by the exceedingly low response rate to our online survey. Low response rates are a common phenomenon, and similar surveys on the topic have had response rates as low as 2.9\% [13]. Web-based surveys are known to have especially low response rates, in part due spam blocking features which prevent the message from ever entering the physician's inbox. When entering into contract with the AMA database, we were advised that their typical response rate was 5\%, and thus, our response rate of $5.2 \%$ was solidly within their normal patterns. This introduces a risk of bias to the data based on what kind of practitioner would be more likely to respond to the survey. In addition, because of the low response rate, our study was likely underpowered to detect differences between the two communities of interest. Nevertheless, our data offer a preliminary assessment of the characteristics of the primary care practices targeted.

In conclusion, while $\mathrm{HBV}$ and $\mathrm{HCC}$ occur at high rates in Chinese and African immigrant communities, in our survey we noted significant gaps in primary care physicians' knowledge of published screening guidelines for these at-risk populations. This study suggests the need for better distribution of available guidelines to physicians practicing in immigrant-rich neighborhoods in order to improve HBV and HCC screening.

\section{Supplementary Material}

Refer to Web version on PubMed Central for supplementary material.

\section{Acknowledgments}

Funding: We gratefully acknowledge that this study was supported by grant 1R03CA164546-01A1 from the National Cancer Institute.

\section{References}

1. El-Serag HB. Epidemiology of viral hepatitis and hepatocellular carcinoma. Gastroenterology. 2012; 142(6):1264-1273. [PubMed: 22537432] 
2. Ferlay J, Shin HR, Bray F, Forman D, Mathers C, Parkin DM. Estimates of worldwide burden of cancer in 2008: GLOBOCAN 2008. Int J Cancer. 2010; 127(12):2893-2917. [PubMed: 21351269]

3. Parkin DM. The global health burden of infection-associated cancers in the year 2002. Int J Cancer. 2006; 118(12):3030-3044. [PubMed: 16404738]

4. Colvin, HM., Mitchell, AE. Hepatitis and Liver Cancer: A National Strategy for Prevention and Control of Hepatitis B and C. The National Academies Press; Washington, D.C.: 2010.

5. Shaughnessy AF. USPSTF Recommendations for Hepatitis B Screening. Am Fam Physician. 2014; 90(11):795.

6. Zhang BH, Yang BH, Tang ZY. Randomized controlled trial of screening for hepatocellular carcinoma. J Cancer Res Clin Oncol. 2004; 130(7):417-422. [PubMed: 15042359]

7. Liaw YF, Sung JJ, Chow WC, Farrell G, Lee CZ, Yuen H, et al. Lamivudine for patients with chronic hepatitis B and advanced liver disease. N Engl J Med. 2004; 351(15):1521-1531. [PubMed: 15470215]

8. Bruix J, Sherman M. Management of hepatocellular carcinoma: an update. Hepatology. 2011; 53(3): 1020-1022. [PubMed: 21374666]

9. Bruix J, Sherman M. Management of hepatocellular carcinoma. Hepatology. 2005; 42(5):12081236. [PubMed: 16250051]

10. Kew MC, Macerollo P. Effect of age on the etiologic role of the hepatitis B virus in hepatocellular carcinoma in blacks. Gastroenterology. 1988; 94(2):439-442. [PubMed: 2446950]

11. Kew MC, Marcus R, Geddes EW. Some characteristics of Mozambican Shangaans with primary hepatocellular cancer. S Afr Med J. 1977; 51(10):306-309. [PubMed: 66757]

12. Bodle EE, Islam N, Kwon SC, Zojwalla N, Ahsan H, Senie RT. Cancer screening practices of Asian American physicians in New York City. J Immigr Minor Health. 2008; 10(3):239-246. [PubMed: 17653863]

13. Chu D, Yang JD, Lok AS, Tran T, Martins EB, Fagan E, et al. Hepatitis B screening and vaccination practices in asian american primary care. Gut Liver. 2013; 7(4):450-457. [PubMed: 23898386]

14. Braillon A. Hepatocellular carcinoma screening among hepatitis B-infected Asian Americans. Journal of Viral Hepatitis. 2013; 20(7):514-514. [PubMed: 23730846]

15. Sarkar M, Stewart S, Yu A, Chen MS, Nguyen TT, Khalili M. Hepatocellular carcinoma screening practices and impact on survival among hepatitis B-infected Asian Americans. Journal of Viral Hepatitis. 2012; 19(8):594-600. [PubMed: 22762144]

16. Khalili M, Guy J, Yu A, Li A, Diamond-Smith N, Stewart S, et al. Hepatitis B and Hepatocellular Carcinoma Screening Among Asian Americans: Survey of Safety Net Healthcare Providers. Digestive Diseases and Sciences. 2011; 56(5):1516-1523. [PubMed: 21046247]

17. Kwon HT, Ma GX, Gold RS, Atkinson NL, Wang MQ. Primary care physicians' cancer screening recommendation practices and perceptions of cancer risk of Asian Americans. Asian Pac J Cancer Prev. 2013; 14(3):1999-2004. [PubMed: 23679307]

18. Adair R, Nwaneri O. Communicable disease in African immigrants in Minneapolis. Arch Intern Med. 1999; 159(1):83-85. [PubMed: 9892335]

19. Ugwu C, Varkey P, Bagniewski S, Lesnick T. Sero-epidemiology of hepatitis B among new refugees to Minnesota. J Immigr Minor Health. 2008; 10(5):469-474. [PubMed: 18066661]

20. Custer B, Sullivan SD, Hazlet TK, Iloeje U, Veenstra DL, Kowdley KV. Global epidemiology of hepatitis B virus. J Clin Gastroenterol. 2004; 38(10 Suppl 3):S158-168. [PubMed: 15602165]

21. Gambino, CP., Trevelyan, EN., Fitzwater, JT. American Community Survey Briefs. U.S. Census Bureau; 2014. The Foreign-Born Population From Africa: 2008-2012. https://www.census.gov/ content/dam/Census/library/publications/2014/acs/acsbr12-16.pdf [Accessed 20 Sep 2016]

22. Grieco, EM., Acosta, YD., De la Cruz, GP., Gambino, C., Gryn, T., Larsen, LJ., Trevelyan, EN., Walters, NP. American Community Survey Reports. U.S. Census Bureau; 2012. The Foreign-Born Population in the United States: 2010. https://www.census.gov/prod/2012pubs/acs-19.pdf [Accessed 20 Sep 2016]

23. New York City Census FactFinder. [Accessed 20 Sep 2016] New York City Department of City Planning: 2010. http://maps.nyc.gov/census/ 
24. McMahon BJ, Bulkow L, Harpster A, Snowball M, Lanier A, Sacco F, et al. Screening for hepatocellular carcinoma in Alaska natives infected with chronic hepatitis B: a 16-year populationbased study. Hepatology. 2000; 32(4 Pt 1):842-846. [PubMed: 11003632]

25. Lai CJ, Nguyen TT, Hwang J, Stewart SL, Kwan A, McPhee SJ. Provider knowledge and practice regarding hepatitis B screening in Chinese-speaking patients. J Cancer Educ. 2007; 22(1):37-41. [PubMed: 17570807]

26. Nguyen TT, Gildengorin G, Truong A, McPhee SJ. Factors influencing physicians' screening behavior for liver cancer among high-risk patients. J Gen Intern Med. 2007; 22(4):523-526. [PubMed: 17372804]

27. Howlader N, NA.Krapcho, M.Neyman, N.Aminou, R.Waldron, W.Altekruse, SF.Kosary, CL.Ruhl, J.Tatalovich, Z.Cho, H.Mariotto, A.Eisner, MP.Lewis, DR.Chen, HS.Feuer, EJ., Cronin, KA., editors. SEER Cancer Statistics Review, 1975-2009 (Vintage 2009 Populations). National Cancer Institute; Bethesda, MD: 2012.

28. Grulich AE, Swerdlow AJ, Head J, Marmot MG. Cancer mortality in African and Caribbean migrants to England and Wales. Br J Cancer. 1992; 66(5):905-911. [PubMed: 1419634]

29. Strong C, Lee S, Tanaka M, Juon HS. Ethnic differences in prevalence and barriers of HBV screening and vaccination among Asian Americans. J Community Health. 2012; 37(5):1071-1080. [PubMed: 22302652]

30. Burman B, Mukhtar N, Toy B, Nguyen T, Chen A, Yu A, et al. Hepatitis B Management in Vulnerable Populations: Gaps in Disease Monitoring and Opportunities for Improved Care. Digestive Diseases and Sciences. 2014; 59(1):46-56. [PubMed: 24052195]

31. Foster T, Hon H, Kanwal F, Han S, Spiegel B. Screening high risk individuals for hepatitis B: physician knowledge, attitudes, and beliefs. Digestive Diseases and Sciences. 2011; 56(12):34713487. [PubMed: 22001940] 


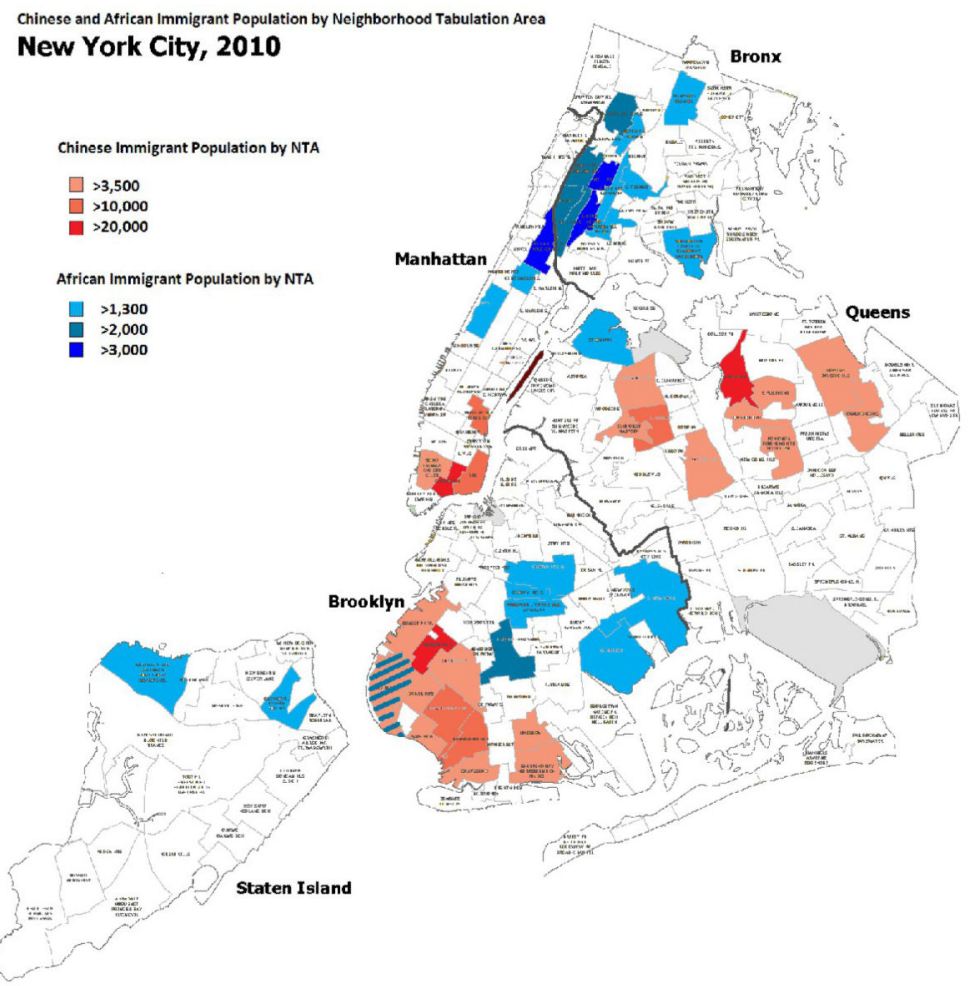

Figure 1.

Distribution of Chinese and African-born individuals by Neighborhood Tabulation Area: New York City, 2010 


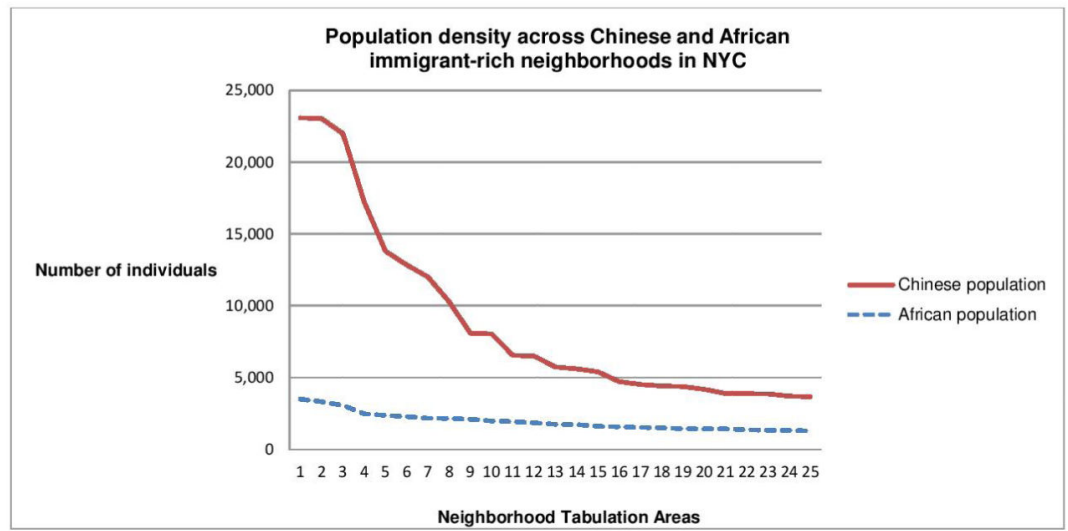

Figure 2.

Population density across Chinese and African immigrant-rich neighborhoods 


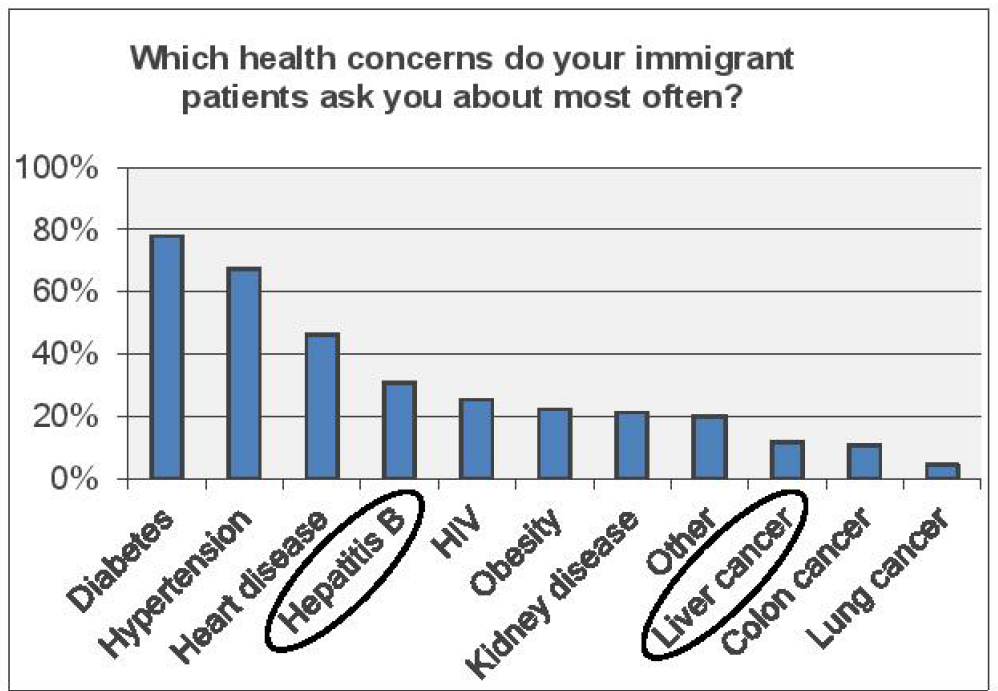

Figure 3.

Most common health concerns of patients, as perceived by providers in immigrant-rich neighborhoods 
Which of the following statements are true?

\begin{tabular}{|l|c|}
\hline & $\begin{array}{c}\text { Correct } \\
\text { response \% }\end{array}$ \\
\hline In patients with hepatitis B, liver cancer only develops if there is cirrhosis (False) & $91.8 \%$ \\
\hline High-risk patients should be screened for liver cancer, even if no symptoms are present (True) & $77.6 \%$ \\
\hline Antiviral therapy for hepatitis B can help prevent liver cancer (True) & $72.9 \%$ \\
\hline Screening for liver cancer involves an ultrasound every 6-12 months (True) & $63.5 \%$ \\
\hline Screening for liver cancer among patients with hepatitis B improves survival (True) & $56.5 \%$ \\
\hline A 25 year-old immigrant from Africa who has hepatitis B should be screened for liver cancer (True) & $54.1 \%$ \\
\hline A 25 year-old immigrant from China who has hepatitis B should be screened for liver cancer (False) & $47.1 \%$ \\
\hline
\end{tabular}

Figure 4.

Knowledge assessment of providers in immigrant-rich communities in New York City 


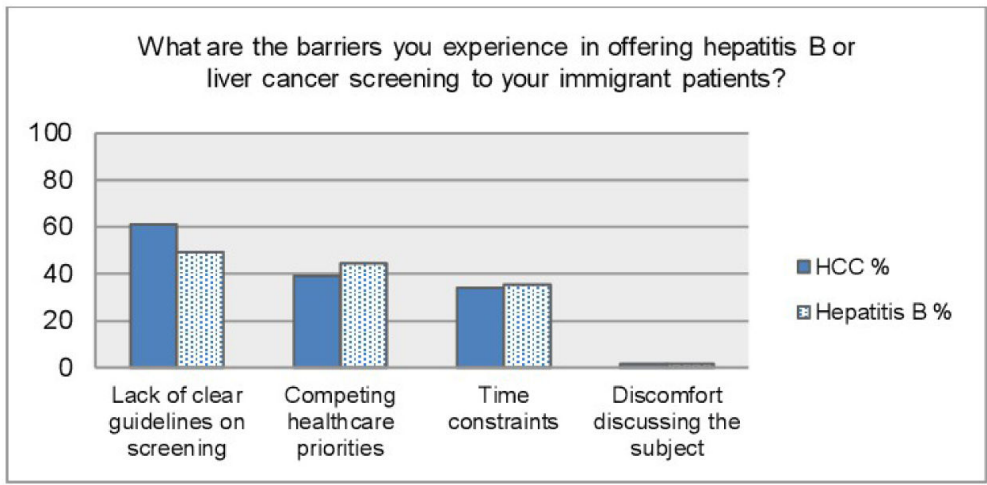

Figure 5.

Barriers to hepatitis B and hepatocellular carcinoma screening, as reported by providers in immigrant-rich communities in New York City 